\title{
La Historia de vita et gestis Scanderbegi Epirotarum Principis (1510) de Marin Barleti: una lectura
}

\author{
The Historia de vita et gestis Scanderbegi Epirotarum Principis \\ (1510) by Marinus Barletius: A Reading \\ Antonio Contreras Martín \\ (Institut d'Estudis Medievals [IEM-UAB])
}

\begin{abstract}
RESUMEN
Jorge Castriota (Gjergj Kastrioti) (c. 1405?-1468), conocido como Scanderbeg, se convierte mediante la Historia de vita et gestis Scanderbegi Epirotarum Principis (1510) de Marin Barleti en un héroe nacional albanés y ejemplo de combatiente cristiano («athleta Christi $)$ ), cuya historia transciende sus fronteras originales y se difunde por Europa. El propósito de este trabajo es un análisis introductorio a fin de observar cómo Marin Barleti confeccionó la biografía de Jorge Castriota, para lo que centraré mi atención en nueve momentos determinantes, que se presentan como ejemplares, en la configuración de la trayetoria vital de protagonista.
\end{abstract}

\section{Palabras Clave}

Scanderbeg, Jorge Castriota, Historia Scanderbegi, Siglo XVI, Marin Barleti.

\section{Abstract}

George Castriota (Gjergj Kastrioti) (c. 1405?-1468) known as Scanderbeg is converted by the Historia de vita et gestis Scanderbegi Epirotarum Principis (1510) by Marinus Barletius into an Albanian national hero and example of a Christian fighter («athleta Christi») whose history transcends its original borders and spreads throughout Europe. The purpose of this work is an introductory analysis in order to observe how Marin Barleti made the biography of George Castriota, for which I will focus my attention on nine determining moments, which are presented as exemplary, in the configuration of the protagonist's life.

\section{KEYWORDS}

Scanderbeg, George Castriota, Historia Scanderbegi, $16^{\text {th }}$ Century, Marinus Barletius.

Recibido: $22 / 02 / 2021$

Aceptado: 16/10/2021 


\section{Preliminar}

Paseo por la colina del Aventino y llego a la Piazza Albania (Roma). Una majestuosa estatua ecuestre, ocupa un lugar destacado. La montura es recia. Su rostro es severo y honorable y está cubierto por una espesa barba. Observo las armas del caballero. Lleva un arnés milanés, una sobreveste y sujeta un alfanje en su mano derecha. Llama la atención su yelmo, desprovisto de visera, coronado por una cimera que es la cabeza de un carnero. Se trata de una representación del noble albanés Jorge Castriota (1940)ํㅣㄹ obra de Romano Romanelli (Fig. 1). Hay otras muy similares en Tirana, Skopie y Pristina.

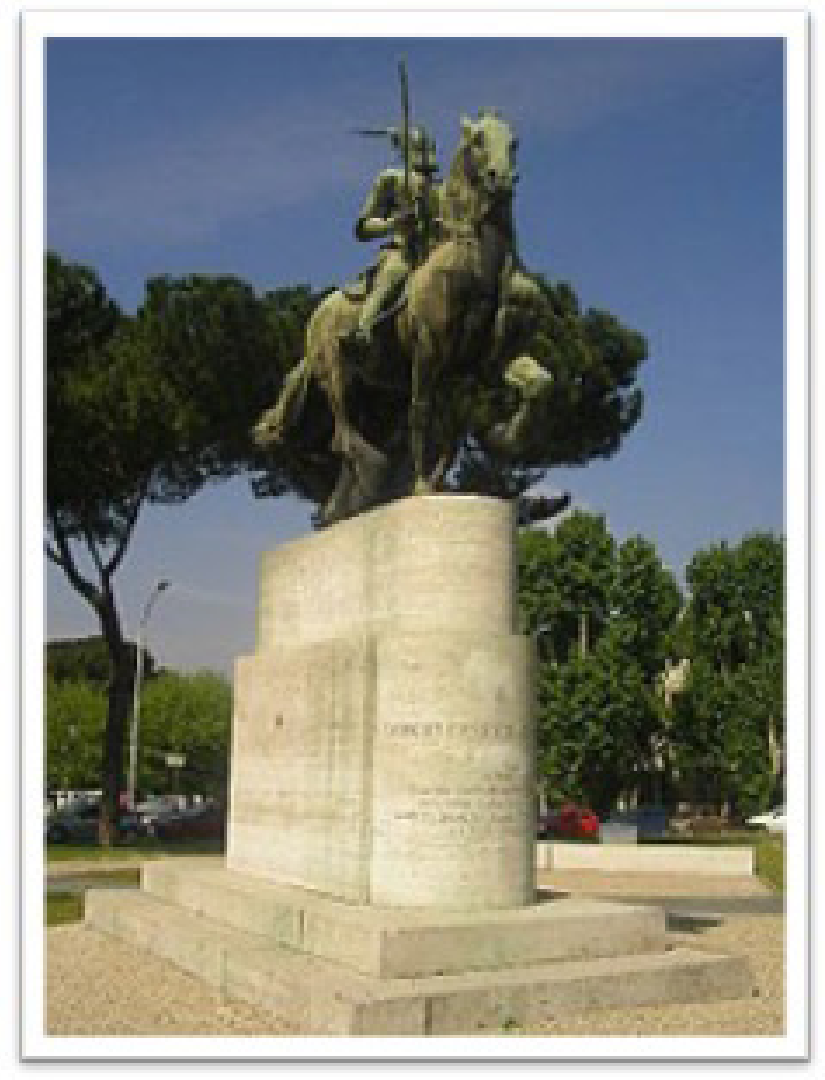

Fig. 1. «Jorge Castriota», de Romano Romanelli (Piazza de Albania, Roma)

En la Galleria degli Uffizi (Florencia), me detengo ante un retrato: "Georgius Scanderbek", obra muy probablemente de Gentile Bellini (Venecia, 1466) ${ }^{2}$. Muestra a un anciano venerable que inspira respeto. Su nariz es aguileña y una larga barba blanca cubre su rostro. Su mirada parece denotar preocupación. Va vestido de rojo, tocado con un sombrero de idéntico color ${ }^{3}$, como muestra de su prestigio y condición (Fig. 2$)^{4}$.

\footnotetext{
1. A partir de ahora, se usarán las formas de los nombres en castellano.

2. Veáse Zeqo (2017).

3. Sobre este color remito a Greenfield (2005).

4. El grabado incluido en Historia de vita et gestis Scanderbegi Epirotarum Principis (1508-1510) (Figura 3), aunque presenta diferencias, guarda estrecha relación con este.
} 


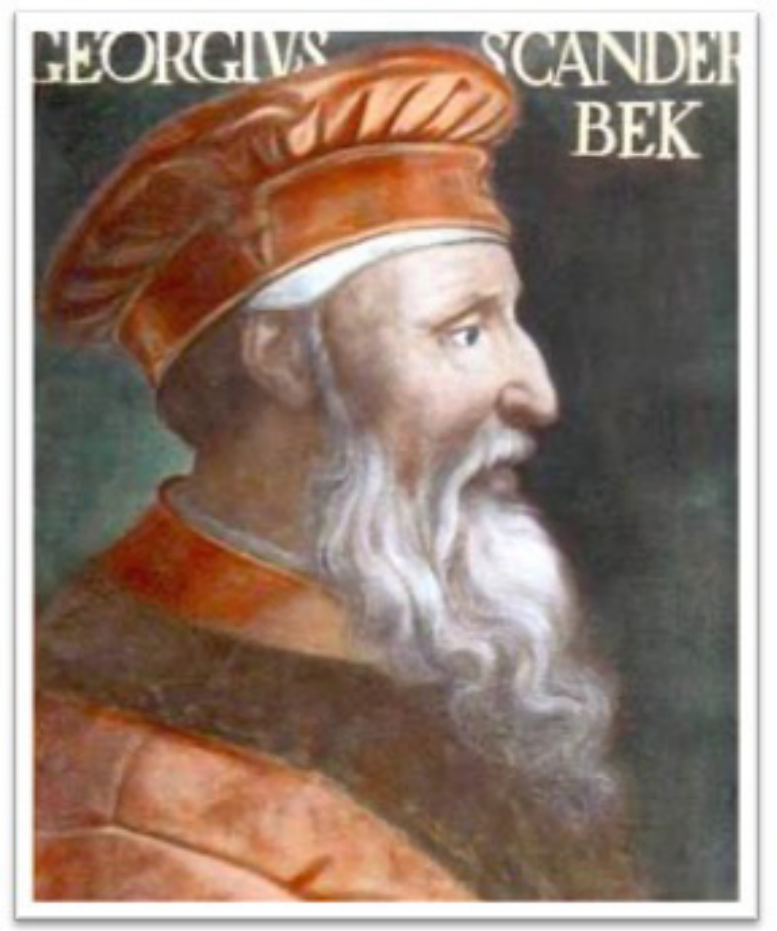

Fig. 2. «Georgius Scanderbek», atribuido a Gentile Bellini. Galleria degli Uffizi (Florencia)

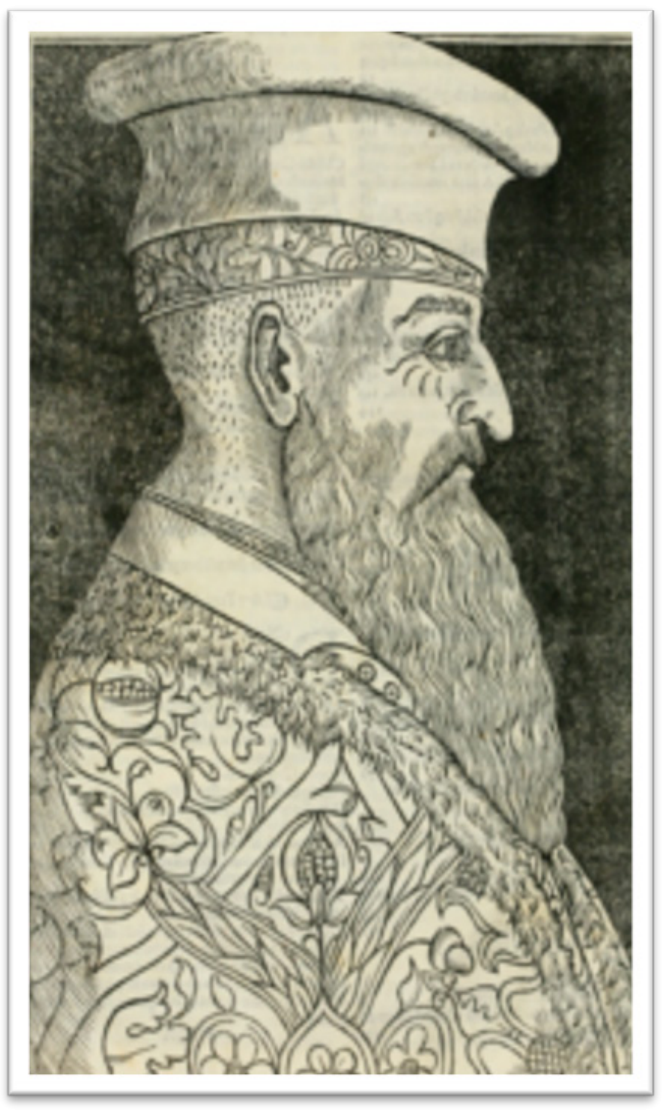

Fig. 3. Jorge Castriota en la Historia de vita et gestis Scanderbegi Epirotarum Principis (1508-1510)

Casi quinientos años separan ambas obras. Una real, la otra imaginada. La segunda, capta a un hombre ya en el final de una intensa vida, un experimentado y prudente gobernante; la primera opta por plasmar a un combatiente entre dos mundos, como revelan sus armas. Dos representaciones, sin embargo, complementarias y que se hallan en consonancia con la imagen del noble albanés elaborada por Marin Barleti en la Historia de vita et gestis Scanderbegi Epirotarum Principis (1508-1510) (Fig. 3) .

\section{La 'biografía heroica' de Jorge Castriota, Scanderbeg ${ }^{6}$}

A la hora de reconstruir la 'biografía' de Jorge Castriota (c. 1405?-1468), pese a la relevancia del personaje y a su enorme actividad desplegada tanto en la Península Balcánica como en tierras italianas, apenas se cuenta con testimonios y las referencias son escasas antes de la obra de Marin Barleti (Marinus Barletius o Marino Barlezio). De él se hace eco de forma muy sucinta, Eneas Silvio Piccolomini en De Europa (1458):

5. Desde ahora HS y se indica el libro y el folio. Para la presentación gráfica de los fragmentos de la obra, al igual que para el resto de obras en latín, se amplían las abreviaturas, se puntúa según las normas actuales y se corrigen los errores.

6. Para su semblanza, véase Noli (1993), Vaccaro (2013) y Brackob (2018); y para un panorama de su proyección, Genesin, Matzinger \& Vallone (2010). 
Georgius Scanderbechius eius haereditatem accepit, nobili loco natus, qui aetatem pene omnem in armis pro Christi nomine pugnando consumpsit, multas et magnas Turcarum turmas bello vicit atque delevit. Solumque quodam modo regionem illam in Evangelio Christi continuit, quamvis hodie toties hostili ferro petita, magna ex parte deserta feratur. Rex Alphonsus saepe milites ira Albaniam misit, et Troiam urbem in potestatem receptam a Turcis defendit ${ }^{7}$. Nepostus, et ad Alphonsum missus, in carcerem coniectus est. Calixtus quoque Romanus pontifex auxilia pecuniaria Scanderbechio non pauca misit [...] Scanderbechio in Albania, multique aliis per Graeciam Turcarum bello laborantibus, pecuniaria subsidia largitus est. (Aeneae Sylvii, 1551: cap. XV, f. 407; y cap. LVIII, f. 461)

Y, también se le menciona en la Kronica Turecka (1481) de Konstantín Michailović de Ostrovica, donde se da noticia breve:

Perfezionato questo armistizio si rivolse contro i principi dell'Albania e uno dopo l'altro li conquistò molto facilmente, perché mentre attacava uno l'altro stava a guardare. Un principe gli oppose resistenza. Si chiamava Skander Ivanović. Fu preso da ragazzo fra i giannizzeri, al tempo dell'imperatore Murad, e osservò tutti gli affari dell'imperatore per tornare alla sua terra, dopo aver conosciuto il favore dell'imperatore. Accade una volta all'imperatore di dire: «Skander, chiedimi il voivodato che vuoi e te lo darò». Egli chiese che gli fosse data la Terra di Ivan, ma non disse che era il figlio di Ivan. L'imperatore glielo concesse ed egli se ne impossessò, ad eccezione delle fortificazioni. Poi riuscì in qualche modo a portare fuori i giannizzeri, che erano nelle fortezze, e le evacuò e se ne impossessò. Dopo l'imperatore Murad attacava continuamente le terre che erano sotto di lui. E anche Maometto, figlio de Murad, dovette lasciarlo in pace fino alla morte, perché risulta facile difendersi dai Turchi per colui che sa le loro disposizioni. (Konstantin Michailović, 2001: cap. XXXIII, 111) ${ }^{8}$

\section{Corrijo la lectura de «Troiam» por «Croiam» (Kruja [Krujë]).}

8. La obra de Giovanni Musachi (Gjon Muzaka) relaciona algunos de los hechos en Breve memoria di li discendenti de casa Musachi. Ahora bien, aunque cercana en el tiempo, se ha demostrado que, sin duda, es posterior a 1514, quizá de1515 (Elsie, 2003: 34). En este relato, Jorge Castriota desempeña un papel determinante en un momento dramático para las tierras albanesas. Su aparición conlleva la esperanza, su desaparición la desolación: «Li Signori giovani d’Albania erano tutti morti quasi; et erano rimasti questi Signori, videlicet: Il Signor Arainiti Comnino, il Signor Coico Bassa, il Signor Nicolò e Paulo Ducaguino, il Signor Ginno Mosachi mio padre, il Sinor Andrea Topia, il Signor Pietro Spani; tutti questi vecchi e li più de loro declinati de forze e de stato; erano cene alcuni figlioli de questi, ma pochi, che per le continue guerre erano consumati, come ho detto; non però ce defendeamo al meglio possibile, benchè haveamo perse assai paese.

Quando poi in tempo del secondo Amuratte arrivò Scanderbegh figlio del donato il padre al detto Amuratt con due altri fratelli tutti piccoli; li due morsero; lui se chiamava Giorgio Castrioto, mà quando se fè Turco, lo chiamarno Scanderbegh, che Scander vol dire Alesandro, e begh vol dir Signor), questo crescendo riuscì. Disposto savio e valente; poi la morte del padre fuggì dal Turco, videlicet mandando il Turco il bassa de Romania contra l'Ungari, mandò anco con lui Scanderbegh, onde il detto bassa fù rotto fugendo il Turchi; il detto Scanderbegh fuggì con l'altri; onde per sorte il cancelliero del bassa se trovò con lui; Scanderbegh lo fè prendere e lo strinse, che le facesse una commissione in nome del Turco al governator de Croya, che le consignasse la terra a lui; alla fine il concelliero la fè benchè recusasse, secondo il suo volere; poi fece morire detto cancelliero, che no lo revelasse. Allora Scanderbeg prese il camino con alcuni Albanesi erano con lui, et arrivato in Albania, et entrato in Croya, disse al governatore l'ordine e lo consegnò à lui: e s’insignorì di Croya terra fortissima; de questo li Signori d’albania n'hebbero gran piacere, e Scanderbegh si fè subito Christiano.

Poi fecero consiglio detti Signori d'Albani in Alessio; chi ce andò personalmente e chi ce mandò suoi sustituti, talchè il detto Scanderbegh fù creato e fetto lor Capitano in Albania, e ciascuni contribuiva de gente e de denari pro rata, et anco alcuni figlioli de quelli signori militavano sotto de lui sì per imprender la guerra, como anco per difendere loro stati; per essere detto Signor prattico alla guera e valente, fù fatto Capitano et ogn'uno l'obedeva.

Et il detto Scanderbegh adomandò per moglie la figlia del Signor Araynati Comnino, lo quale Signor lo mandò a consultar con lo Signor Ginno mio padre; che il detto Signor Arayniti era cognato a mio padre, perchè hebbe per moglie la Signora Maria Mosachj sorella de mio padre, com'ho detto, per il chè detto matrimonio fù concluso, e prese la Signora Andronica Comminata per moglie, quale era 
A diferencia de estas breves referencias, Marin Barleti confecciona una extensa obra, cuyo título Historia de vita et gestis Scanderbegi Epirotarum Principis, ya denota su objetivo'. Deja claro que debe entenderse como la relación «verídica” («Historia») no tan sólo de la 'vida' («vita»), sino también de los 'hechos de armas' («gestis») del personaje, a quien menciona tan sólo con su célebre sobrenombre «Scanderbeg», y al que nombra como «Príncipe de Épiro». Barleti la dedica a Ferrante Castriota Scanderbeg, duque de San Pietro in Galantina, nieto del protagonista («ad Don Ferandum Castriotum eius nepotem», HS, fol. $\mathrm{I}^{\mathrm{r}}$ ), y la compone movido por el afán de redactar una 'biografía' con la que revindicar la figura de este glorioso guerrero, del que, sin embargo, nadie se había ocupado hasta ese momento. Como Barleti afirma, debido a la ausencia de un corpus escrito debe recurrir a 'fuentes orales', proporcionadas, por un lado, por testimonios de testigos oculares merecedores de crédito ( $«$ Non commenta in iis sed a maioribus diligenter relata et a quibusdam etiam, qui interferunt visa memoriae mandavi, fidemque plurimorum et veracium virorum, non levem tantum modo et sutilem traditionem sum secutus $\gg, H S$, Praefatio, f. II $^{\mathrm{r}}$ ), y, por el otro, por la tradición oral albanesa, en la que ya circulaban composiciones sobre el héroe, como recoge Marco Antonio Sabellico en Historia rerum Venetarum ab urbe condita, libri XXXIII (Venecia, 1487):

Quo factum esse aiunt, ut post eius mortem eximia viri admiratione populi imbuti, quasi in illo aliquid videssent quod humanum excederet fastigium: grati principis memoriam solemnibus consecrarint carminibus. Retulerunt mihi fide digni viri, vel medio ardore belli, et tum quum Barbarorum ${ }^{10}$ armis omnia strepebant, puellarum coetus in his urbibus quibus ille imperavit octavo quoque die mediis triviis core solitos, ac defuncti principis (ut veteres magnorum heroum in conviis solebant) laudes decantare. (Sabellicii, 1556: Decadis Tertiae, Liber Ix, ff. 922-923)

Barleti articula la narración a partir de trece libros de extensión y contenido diversos (Liber Primus, ff. II $^{\mathrm{r}}$-XIIII' ${ }^{\mathrm{r}}$; Liber Secundus, ff. XIIII'-XXVIII' ${ }^{\mathrm{r}}$; Liber Tertius, ff. XXVIII' ${ }^{\mathrm{v}}$-XL ${ }^{\mathrm{v}}$; Liber Quartus, ff. XLI ${ }^{\mathrm{r}}-\mathrm{LII}^{\mathrm{v}}$; Liber Quintus, ff. LII $-\mathrm{LXV}^{\mathrm{v}}$; Liber Sextus, ff. LXV ${ }^{\mathrm{v}}-\mathrm{LXXXIV}^{\mathrm{r}}$; Liber Septimus, ff. LXXXIV ${ }^{\mathrm{v}}$-XCVIII ${ }^{\mathrm{r}}$; Liber Otavus, ff. XCVIII ${ }^{\mathrm{r}}-\mathrm{CIX}^{\mathrm{r}}$; Liber Nonus, ff. CIX $^{\mathrm{r}}-\mathrm{CXXI}^{\mathrm{r}}$; Liber Decimus CXXII $^{\mathrm{r}}-\mathrm{CXXXII}^{\mathrm{v}}$; Liber Undecimus, ff. CXXXII ${ }^{\mathrm{v}}-\mathrm{CLI}^{\mathrm{r}}$; Liber Duodecimus, ff. CLI ${ }^{\mathrm{r}}-\mathrm{CLV}^{\mathrm{v}}$; y Liber Tertiusdecimus, ff. $\mathrm{CLVI}^{\mathrm{r}}-\mathrm{CLIX}^{\mathrm{v}}$ ), a los que precede un índice ( $\ll$ Index per capita eorum que in iis libris continentur $\gg$ ) y un Prólogo-dedicatoria («Praefatio», ff. $\left.\mathrm{I}^{\mathrm{r}}-\mathrm{II}^{\mathrm{r}}\right)$.

Ahora bien, de entre todos los «momentos» narrados, destacan por su relevancia diez que deben entenderse como «ejemplares» en la configuración de la trayectoria vital del protagonista: el linaje y la familia, el vaticinio prodigioso, la señal heroica y la infancia, el cambio onomástico,

mia sorella consoprina, et hebbe nome poi Scanderbegha, per il nome del marito. Et finchè visse detto Signor, sempre con sua virtù e valore et ajuto de quelli Signorir fè gran stragge de' Turchi con molte vittorie non senza morte de' nostri Signori e cavalieri.

Mà al fine crescendo le forze del Turco, et havendo preso la Bulgaria, la Servia, la Bosna, la Morea e tutt'il resto de quelli paesi, non possevamo più resistere.

Ce prese anco a noi la Musachia e Belgrado capo de Musacchia detta; ma mio padre morse poco avante, oltre la vecchiezza, de dispiacere, e cossi anco il Signor Arayniti Commnino suo cognato.

Il Signor Scanderbegh vedendosi anco lui affannato da inimici con poca speranza, lassaltò la febre in Alexio, e li morse nel 1466 nell’anno de sua età 63. Possete considerare, qual restò quel paese, havendo perso un tal capitano; pochi figliuoli de' Signori erano remasti che non fossero morti in sì lunghe e crudeli guerre; li vecchi erano già quasi tutti morti sì per l'età come per il dolore» (Hopf, 1873: 274-275).

9. Sobre Marin Barleti, su obra y el entorno veneciano en que se produce la publicación, remito a Nadin (2012).

10. Es decir, 'turcos'. 
la formación y los primeros hechos de armas, la prosopografía, el retorno al hogar, la enfermedad y la muerte, la profanación de la tumba y del cuerpo, y la muerte de su caballo. Aquí me centraré sólo en nueve ${ }^{11}$.

\subsection{El linaje y la familia}

Barleti fija con escrúpulo la estirpe de Jorge Castriota. En él confluyen la sangre albanesa del padre, Juan Castriota [Gjon Kastrioti], y la serbia de la madre, Vojsova Tribalda o Tripalda, una noble de la región de Polog, emparentada con la dinastía Branković:

Per id tempus ne nimis alte exordiendo alienas historias percensere velle videar in Epiro inter caeteros regulos, principesque satis nobile nomen Ioannis Castrioti, tum caeterarum urbium tum Croiae praecipue imperio erat. Et quia neque latere penitus Scanderbegi genus aequum duco, neque omnem avorum eius seriem perscribere in animo est, illud unum attigisse contentus ero, auctores gentis Castriotae ex Aemathia nobili ortu fluxisse, imperitasseque, pari gloria fortunaque in Epiro, eos omnes (ut iam orsus sum) Ioannes prudentia, gravitate, ac animi invicti magnitudine, coeterisque deinceps virtutibus, atque egregio etiam (si quid ad rem facit) corporis decore facile superavit, uxori Voisavae nomen erat, non indignam eo viro tum pater nobilissimus Tribalorum princeps, tum forma moresque ac insignis super muliebre ingenium animus faciebat. $\left(H S, \mathrm{I}, \mathrm{f} . \mathrm{II}^{\mathrm{r}-\mathrm{v}}\right)^{12}$

Y ofrece los nombres de sus tres hermanos: «Reposium [...] Stanisam, Constantinum» (Reposh, Stanisha, Konstandin); y de sus cinco hermanas: «Maram, Iellam, Angelinam, Vlaicam, Mamizam» (Mara, Jelena o Jela, Angelina, Vlajka y Mamica), sin duda con el fin de que los descendientes pudieran conocer su parentesco.

\subsection{El vaticinio prodigioso}

Barleti incluye el perturbador 'sueño de Vojsava', rasgo característico de la literatura heroica (Campbell, 1949), con el que se anuncia el nacimiento de un individuo prodigioso dotado de excepcionales cualidades:

Sed antequam progrediar ulterius omittendum non censeo quod vel prodigiis ipsis ostensum et a plaerisque vaticinatum de hominis gloria accepi. Licet id antiquis simillimum fabulis aspernaturos plurimos non ignorem. Ferunt namque quum concepisset eum mater, serpentem se enixam eius magnitudinis somniavit, qui totam ferme operiens Epirum caput inter Turcarum fines protenderet et cruentis faucibus eos absorberet, caudam vero teneret in mari inter Christianos, et Veneti maxime imperii términos. (HS, I, f. II ${ }^{\mathrm{v}}$ )

y, a continuación, revela su significado:

narratum id sibi, Ioannes, quum non eius generis res esset, quae extis ullis scrutaretur, vel Apollinem exigeret interpretem, ingenti laetitia solatus uxorem, naciturum ex ea virum ominatus facile est, clarum bello, factisque, Qui Turcarum simul hostis acerrimus idem-

11. Excluyo el análisis sobre la muerte del caballo, pues me ocuparé del tema en otro lugar.

12. Paolo Angelo (1539), deudor de Barleti, refiere que: «Et la matre di esso Scand. chiamata Voisaua, fu figliola del Signore di Pollogo, che è una parte della Macedonia \& Bulgaria» (Laporta, 2004: 4). 
que, dux foelicissimus et Christianae fidei propugnator, ac Veneti cultor imperii perpetuus existeret. ( $H S$, I, f. $\mathrm{II}^{\mathrm{v}}$ )

Ahora bien, ¿̇cómo dilucidar el significado de la «serpiente» («serpentem»), dado que para la Iglesia cristiana su naturaleza posee una ambivalencia simbólica positivo-negativa? La interpretación paterna, según la cual se convertirá en «dux foelicissimus et Christianae fidei propugnator $\gg$, se decanta claramente por su valor simbólico positivo ${ }^{13} \cdot$ ¿Cómo es posible? En primer lugar, hay que tener en cuenta que la imagen de una gigantesca serpiente que amenaza con sus fauces con devorar al Imperio Otomano, un enemigo muy poderoso y superior («serpentem se enixam eius magnitudinis somniavit, qui totam ferme operiens Epirum caput inter Turcarum fines protenderet et cruentis faucibus eos absorberet, caudam vero teneret in mari inter Christianos, et Veneti maxime imperii terminos»), parece remitir y emparentarse con el episodio del Éxodo bíblico, en el que el cayado de Aarón, hermano de Moisés, se transforma en este reptil y engulle al grupo de serpientes, en que se habían metamorfoseado los cayados de los sacerdotes egipcios, con lo que se pone de manifiesto la superioridad de los hebreos, al hallarse bajo la protección de Dios ${ }^{14}$, al igual que lo estarían los cristianos albaneses con la figura de Jorge Castriota, adalid de Dios, con respecto de los turcos musulmanes. En segundo lugar, debería considerarse que Barleti parece emplear el término como sinónimo de 'draco' (dragón), confusión lingüística heredada desde la Antigüedad y que pervive en los bestiarios medievales (Malaxecheverría, $1987)^{15}$; y, en tercer lugar, que el autor descarta atribuirle cualquier contenido negativo ${ }^{16}$, con lo que se inscribiría en una corriente en la que el 'dragón' se usa como símbolo de entrega cristiana y de devoción por San Jorge, como se documenta entre los reyes de la casa de Aragón, quienes a partir de Pedro IV el Ceremonioso incluirán en su cimera un 'dragón alado' (Montaner Frutos, 2013), o entre los miembros de la Orden del Dragón (Societas Draconistarum), instituida por el rey Segismundo de Hungría (1408), continuadora de la Orden de San Jorge (Societas Militiae Sancti Georgii), fundada por Carlos de Hungría (1318), cuyo emblema era la «cruz y el dragón» (Boulton, 1987: 348-355) 17. $^{17}$

13. Como recuerda Charbonneau-Lassay, haciéndose eco de las palabras de René Guénon, llegó a identificarse con Cristo («Même dans l'iconographie chrétienne, le serpent est parfois un symbole du Christ», 2006: 772). Pero, también posee connotaciones negativas y se considera la imagen del Diablo, como muestran, por ejemplo, las palabras de San Juan: «Et apprehendit draconem, serpentem antiquum, qui est Diabolus et Satanas, et ligavit eum per annos mille», en Apocalysis Iohannis, 20, 2 (Colunga \& Turrado, 1953). Para su análisis como símbolo diabólico, remito a Russell (1984).

14. «Dixitque Dominus ad Moysen et Aaron: Cum dixerit vobis Pharao, Ostendite signa: dices ad Aaron: Tolle virgam tuam, et projice eam coram Pharaone, ac vertetur in colubrum. Ingressi itaque Moyses et Aaron ad Pharaonem, fecerunt sicut praeceperat Dominus: tulitque Aaron virgam coram Pharaone et servis ejus, quae versa est in colubrum. Vocavit autem Pharao sapientes et maleficos: et fecerunt etiam ipsi per incantationes aegyptiacas et arcana quaedam similiter. Projeceruntque singuli virgas suas, quae versae sunt in dracones: sed devoravit virga Aaron virgas eorum» Exodus, 7, 8-12 (Colunga \& Turrado, 1953).

15. Así, por ejemplo, en el Bestiario de Cambridge se lee: «El dragón es la mayor de todas las serpientes» (Malaxecheverría, 1986: 180). 16. No se trataría de un caso único, puesto que ya en el Phisiologus (siglos II al V) se destacan elementos positivos de ella. Véase, Il Fisiologo (Zambon, 1982: 49-50) y El Fisiólogo (Guglielmi, 2002: 79-80).

17. De igual modo, si se considera que durante la Edad Media y el Renacimiento se emplea la serpiente como emblema con la apariencia de una «G» mayúscula, y que llega interpretarse incluso como la inicial de un nombre o apellido (Charbonneau-Lassay, 2006: 780-781), cabría plantearse si la imagen de esa serpiente, que podría representarse mentalmente en posición arqueada y con la cabeza amenazante, como si fuese a atacar, no podría identificarse con la inicial del nombre de protagonista: Gjergj. 


\subsection{La señal heroica y la infancia}

Jorge Castriota, según Barleti, nace con una señal en el brazo derecho, que pone de manifiesto, su destino heroico:

Nihil fefellit se, vel alios sollicitus parens, sed aucta magis est vaticinio fides puero nato, nanque aliud pulchrius longe clariusque bellici decoris signum in dextero infantis brachio visum forma, ensis ita expressa, ut manu facta videretur. $\mathrm{O}$ insignem naturae liberalitatem, vel nascentibus nobis subiicit documenta et in corpore ipso figit insignia, quae postmodum sint sequenda. (HS, I, f. II ${ }^{\mathrm{v}}$ )

Una 'espada' («ensis»). A la 'espada' se le dota de un significado, que se desliza de lo real a lo figurado, ya desde el Nuevo Testamento, donde se identifica con el «Espíritu» y simboliza «la palabra de Dios» («et galeam salutis assumite et gladium Spiritus, quod est verbum Dei», Epistula Sancti Pauli Apostoli ad Ephesios, 6, 17, Colunga \& Turrado, 1953). Asimismo, ya desde el siglo XIII, en el imaginario de la caballería, además de ser el arma ofensiva propia de un caballero, se le confiere un doble significado (Flori, 1986), que se extenderá incluso a cada una de sus partes, al igual que al resto de los elementos que componen el arnés del caballero (Riquer, 2011); y, así, por ejemplo, simbolizará la «obediencia» en el Lancelot en prose (1215-1225) (Micha, 1978-1982: VIIII, 250-252) o la «justicia» en el Llibre de l'orde de cavalleria (1276) de Ramon Llull (Llull, 1988: 201) o en el poema L'arnès del cavaller (1370-1380) de Pere March (March, 1993: vv. 676$679,219)$. Ambas virtudes se darán claramente en el personaje.

A la infancia (infantia) en la Edad Media, generalmente, se le dedica poco interés, fundamentalmente, porque esta etapa de la vida se percibe como un preámbulo necesario por el que se debe pasar antes de llegar a la pubertad (pueritia), a la adolescencia (adolescentia) y, posterioremente, a la juventud (iuventus), que es cuando se despliega el potencial del ser humano (Burrow, 1986). La vida de este joven, que se desarrolla como la de otros nobles de la época (Orme, 1984; Riché \& Alexandre-Bidon, 1994), está marcada por su destino como defensor de la fe cristiana:

Infans deinde protinus cum passa est aetas, ad arma et arcus reptavit, atque in ipsis puerilis aetatis rudimentis id specimen praebuit sui, ut satis cuique futurae virtutis dilucerent indicia, is post hac in dies visebatur in adolescente vitae cultus, ea morum elegantia et Christianae fidei promissa religio quaedam, ut omnium in se ora oculosque converteret. Unum parentes, unum fratres, unum caeteri illius regionis homines cum tacita admiratione suspiciebant, de eo omnes sermones et laeta ubique vulgi murmura erant, velut rarum illud ea aetas specimen protulisset. Nam ad ea praesagia naturae gratia quoque quaedam corporisque species, quae condit omnia non deerat. Sed non ex tam foelici (ut ita dixerim) palmite fructus ullos colligere sollicitis parentum votis, fata concessere. (HS, I, f. $\mathrm{II}^{\mathrm{v}}$ )

Sin embargo, un suceso alterará su existencia, pues él y sus hermanos deberán desplazarse como rehenes a la corte del sultán Mehmed I, donde será obligado a renunciar al cristianismo ortodoxo y a convertirse al Islam, tras practicársele la circunscisión:

$[\ldots]$ datus proinde cum fratribus Georgius est, qui prae caeteris nimis multum secum privati, publicique luctus tulit. Sed non ideo patrem concepta iampridem de filio spes deseruit, multis oculis amplexibusque, in eum praecipue prolapsus, ac deorum fidem appellans, ne inanem expectationem suam redderent, a se dimisit. Atque aliquis ex vulgo protensam vitam virilesque annos precatus puero domesticum sibi hostem incendiumque educaturum Amurathem dixit. Deducti ad Ottomanum adolescentuli primam perfidiam 
eius in eo sensere. Quod abiecta, quae eorum parentibus praestita erat, fide, circumcidi Macometano ritu illico iussi sunt. $\left(H S, \mathrm{I}, \mathrm{f} . \mathrm{III}^{\mathrm{r}}\right)^{18}$

\subsection{El cambio onomástico}

Durante su cautiverio, Jorge Castriota cambia de nombre y pasa a ser conocido como 'Scanderbeg':

Nunc ad propositum revertar, Epirensibus varia nomina prioribus mutatis indita sunt, ex consuetudine gentis. Georgius, seu casu, seu quia nobilior inter caeteros indoles egregrium nescio quod prae se ferre videbatur Scanderbeg appellatus. Idem quod apud nos Alexander dominus. $\left(H S, \mathrm{I}, \mathrm{f} . \mathrm{III}^{\mathrm{r}}\right)$

¿Cómo explicarlo? Barleti se limita a recoger el dato y a ofrecer el significado del término «Scanderbeg» (Iskander Beğ), a saber, «Alexander dominus», es decir, 'Señor Alejandro', equivalente a la forma 'Mi señor Alejandro'. Se ha propuesto que la sustitución de 'Jorge', un nombre con profundas connotaciones cristianas, al ser el propio de un santo guerrero, por 'Alejandro' (o 'Leka'), de uso frecuente entre los albaneses, podría justificarse, por un lado, como una muestra de su alejamiento de la antigua fe, y, por el otro, como un acto de demostración de descender de los antiguos ilirios y por extensión de Alejandro Magno ${ }^{19}$. Ahora bien, es necesario subrayar que el cambio de un nombre es un hecho determinante en la vida de un noble, ya que supone abandonar su existencia anterior. La adopción voluntaria u otorgada implica el deseo de identificarse con el portador del mismo y, por tanto, asumir su 'biografía' ${ }^{20}$. De ese modo, Jorge Castriota al convertirse en «Scanderbeg» (Iskander Beğ) («Alexander dominus») pretendería devenir émulo del rey Alejandro Magno, que gozaba de un gran prestigio en el Medievo (Stock, 2016).

\subsection{La formación y los primeros hechos de armas}

El período de formación de un caballero (miles) es el que configurará, y en el fondo, condicionará su posterior existencia (Keen, 1984). Scanderbeg recibe en la Escuela de Palacio una cuidada educación y un esmerado adiestramiento en el arte de la guerra, y se convierte en un notable guerrero:

Decreta sunt eis copiosa, honorificaque alimenta, sed Scanderbegum praecipuae cum indoles ipsa, tum aetas (vixdum enim nonum attigerat annum) Tyranno conciliabat. Praeceptores (ut aptior est ea ad discendum aetas) varii generis non negati. Brevi itaque temporis spatio promptum ad omnia ingenium linguam litterasque Turcaicas, Arabicas, Graecas, Italicas Illyricas didicit. Crescentibus vero paulatim cum aetate viribus adolescentem illa unica incessit libido, nullum sane genus bellicae virtutis intentatum transire, ac nunc gladio, nunc arcu, iaculoque tum eques, tum pedes, incredibilia prope omnia promptissimi corporis experimenta edere, audere aliquid, semper maius annis. Et prius-

18. El traslado de los hijos de Juan Castriota y de otros nobles albaneses se produjo en 1415, tras una victoriosa campaña de este sultán, según relata Kritovoulos en su Historia de Mehmet el Conquistador (1954).

19. Asimismo, Hodgkinson (2004: 59-60) propone además que la adopción de este nombre pudo ser percibida por los turcos con ironía, ya que todos los albaneses pretendían descender del macedonio.

20. Un caso notorio es el de Carlos IV de Bohemia (1316-1378). Véase Contreras Martín (2017). 
quam vis ipsa membrorum, ferocia aequaret animi, ac nondum pares bellicis sudoribus spiritus viderentur, amor fuit vario certaminum genere, aequales quotidie provocando crebris spectaculis favorem sibi apud omnium animos principisque praecipue ipsius conciliare, et semper aliquid ferre laudis, ac iam inde viam ad futurum decus et alimenta ad famam praestruere. Mox ingentibus lacertis confirmata iam aetate ardua quaeque fortissimi viri munia obire pati famem sitim, algorem, aestus, vigilias, quibus ex rebus assidua exercitatione optima profecto se ad omnem militiam brevi praestitit. (HS, I, f. III")

Un miembro de la Sipâhi, un silahtars ${ }^{21}$, que será nombrado 'comandante' («Sanzachi») y enviado a combatir a la frontera asiática (Stein, 2007):

Quare non passus Amurathes diutius privatum eum apud se versari, Sanzachi insignibus primum decoravit. Gradus autem is est dignitatis, apud Turcas secundus post Bassas, maior tamen minorque auctoritas inest officio prout latius ei ius liberalitas ipsa principis tribuit. Scanderbeg igitur tunc quinque equitum milibus praepositus fuit. Caeteri quoque fratres iisdem ornati dignitatibus, atque ad varia munia et bellorum necessitates in diversas partes missi satis nominis quotidie promerebantur. Caeterum is non multo post vixdum octavum et decimum egressum annum in Asiam iussum Tyranni profectus. (HS, $\left.\mathrm{I}, \mathrm{f} . \mathrm{I}^{\mathrm{v}}\right)$

Allí demostrará comportarse con mesura, y sobresaldrá por su «prudencia», como cabe esperar de un líder:

Admirata magis ferme prudentia eius est ab Amurathe quam rerum tam foelix succesus. Quum is non solius laus, sed cum fortuna quoque communis esset. Prudentiae vero atque consilii fructus solum sibi ingenium vendicaret. $(H S, \mathrm{I}, \mathrm{f} . \mathrm{IIII})^{22}$

\subsection{La prosopografía}

Sabido es que la prosopografía de un héroe, junto con su etopeya, es esencial para elaborar su retrato, con el que se proporcionará una imagen en consonancia con sus hechos, que contribuirá a enaltecerlo, tal y como recomendaban desde la Antigüedad los tratados de fisiognomía. Ahora bien, si se exceptúa el retrato atribuido a Bellini, que muestra a Scanderbeg ya en su senectud, no se cuenta con más descripción que la ofrecida por Barleti. Si bien el autor describe las diferentes cualidades de las que está dotado el protagonista, tan sólo se ofrece una breve descripción física del mismo en su juventud:

Deposita ocius ambo veste, ultimisque tunicis, nudi prosilientes in medium novo genere spectaculi omnium oculos hausere. Et quamvis fiduciam ingentem vultu, verbisque ostenderet Castriotus, ingens tamen cura mixto dolore spectantium animos incessit. Et simul aetas simul pulchritudo ipsa corporis, ac decor membrorum subiectus oculis multum et pietatis et favoris excitaverant. Statura celsa, proceraque, brachia in homine pulchriora non visa, collum robustum obstippumque, ut laudatur in athletis, humerorum mira latitudo, color candidus, latenti velut purpura suffusus. Aspectus oculorum non torvus, non

\footnotetext{
21. La Sipâhi (caballería pesada) estaba integrada por miembros pertenecientes a la alta nobleza turca (sipâhi) y por guerreros elegidos (silahtars), cuidadosamente seleccionados entre los más destacados del Imperio Otomano. Los jinetes llevaban un arnés compuesto por cota de malla de láminas, rodela, arco compuesto, lanza, maza y hacha. Véase Seadlar (2015).

22. Scanderbeg dará muestras de su capacidad de adaptarse a las condiciones orográficas y aprovecharlas, como hará después durante su defensa de Albania. Véase Isaku (1968).
} 
vanus, sed gratissimus. Adiuvabant haec multum caeteras virtutes atque animi bona augere videbantur. $\left(H S\right.$, I, f. IIII $\left.{ }^{\mathrm{r}}\right)$

De ella se observa que ofrece una imagen ajustada al canon de belleza clásico, imperante en la época, basado en los principios de proporción y armonía: su elevada estatura, la proporción de sus miembros, la blancura de su piel y la expresión de sus ojos de mirada pura, así lo revelan ${ }^{23}$.

\subsection{El retorno al hogar}

El regreso al hogar es un suceso transcendente, pues en él convergen los tres elementos que articularán desde ese momento su vida: el abandono de la órbita turca al desnaturarse del sultán Murad II, la vuelta al cristianismo y la lucha por la libertad de las tierras albanesas por medio de la unión (Liga de Lezhë). Barleti recurre a un emotivo discurso, muestra de la capacidad elocutiva de Scanderbeg ${ }^{24}$, en el que éste expresa la sinceridad de sus intenciones, apela a la unidad y reclama para sí la fidelidad de la nobleza, con el fin de preservar la libertad albanesa:

Neque novum neque inopinatum quippiam hic video duces ac milites fortissimi quod non iampridem animus meus praesagierit et sibi promiserit quum et veterem gentis nobilitatem et publica vestra ac privata in Ioannem patrem officia mecum sedulus repeterem. De me enim (verbo absit adrastia) nullo unquam vitae tempore dubitavi quum hanc mentem hanc pietatem in patriam servarem hos gererem pro libertate spiritus eadem vestra quoque desideria paria mecum vota erant ut saepius (id quod libenter memini et nunc audacter refero) degentem apud Tyrannum omnibus studiis atque officiis ad hoc muneris invitastis oblitus fortasse tum patriae oblitus mei decoris et immemor prorsus libertatis visus sum vobis quum nulla satis certa spe... $\left(H S, \mathrm{I}, \mathrm{f} . \mathrm{X}^{\mathrm{r}}\right)^{25}$

\subsection{La enfermedad y la muerte}

Los últimos días de vida ponen de manifiesto su intento desesperado por consolidar una alianza, que la muerte, causada por la malaria, trunca:

et Lyssum tandem pervenisset (ea enim urbs Veneto imperio parens Scanderbego peculiaris, et percara erat) ut ibi socios et confoederatos ad concilium congregaret, in gravem incidit febrem/ Unde morbo in dies invalescente magis timens sibi et iam extremum vitae tempus adventasse ratus omnes socios et principes, qui ad eum venerant convocari iussit. $\left(H S\right.$, XIII, f. CLVII $\left.{ }^{\mathrm{r}}\right)$

Tiene la conciencia tranquila, pues ha vivido como un buen cristiano y morirá como tal. De ahí que Barleti describa, aunque brevemente, los pasos que conducen a la muerte conforme al

23. Como se plasma ejemplarmente en el David (1504) de Miguel Ángel o en el Hombre de Vitrubio (hacia 1490) de Leonardo da Vinci.

24. Como después se menciona: «Omnesque sic benigne alloquutus est» (HS, XIII, f. CLVII $)^{r}$.

25. Scanderbeg y sus hombres, así como el pueblo albanés, no combaten por razones religiosas, por lo que su actitud no debe entenderse como una 'cruzada', sino que lo hacen para defender su modo de vida y para conservar su patrimonio y sus tierras. Kritovoulos destaca la oposición denodada de los albaneses a las imposiciones turcas: «[The Albanian were] determined to be autonomous and free in every way, and were unwilling to pay a yearly tax, as did all their neighbors, or to furnish troops for expeditions, either to the Sultan's father or to the Sultan himself, or to obey him at all», (Kritovoulos, 1954: V, 61, 210-211). Se refiere respectivamente a Murad II y a Mehmet II. 
ceremonial recogido en los manuales del «ars bene moriendi» (Sanmartín, 2006) y su posterior entierro, según los usos del momento. Así, rodeado de sus aliados y parientes, se confiesa, comulga y muere:

Scanderbegus vero eadem nocte qua Turcaicus et barbarus exercitus aufugit post sacram confessionis poenitentiam caeteraque ecclesiastica sacramenta qua decet veneratione percepta continue se animumque suum Deo Maximo tradens decimosexto Kalendas Februarias Anno Domini MCCCCLVI extremum diem suum obiit fertur. Igitur Scanderbegus tres et LX annos natus. XXIIII sui imperii anno de vita migrasse/ Auguratus est autem Scanderbegus prima auspicia imperii sui quarto Kalendas Decembris anno eiusdem Domini CCCC.XLIII. (HS, XIII, f. CLIX ${ }^{\mathrm{rv}}$ )

A continuación, se le entierra honrosamente:

Sepultus autem est Scanderbegus in urbe Lyssi in ecclesia maiori divi Nicolai cuius exequiae et feriae more maiorum ingenti pompa luctuque militum suorum et omnium principum et ducum dolore maximo patrio ritu deductae sunt, eoque loco ossa eius reposita in pace quieverunt. (HS, XIII, f. CLIX v $)^{26}$

\subsection{La profanación de la tumba y del cuerpo}

La tumba de un héroe se convierte en lugar de recuerdo, un espacio de la memoria, una 'historia en piedra', (Schama, 1995) que debe protegerse y que deviene, a menudo, el «axis mundi» de un pueblo (Tuan, 1974). Barleti concluye la historia con el ominoso suceso de la profanación de la tumba y del cuerpo del príncipe, tras la caída de Lezhë:

Quo tempore Turcae et barbari potiti urbe Lyssi corpus Scanderbegi summo desiderio repertum de tumulo extraxerunt et quem tantopere vivum reformidabant et ad eius tantum auditum nomen fugiebant, mortuum et iam dissolutum (nescio an id divina dispensatione factum sit) videre summopere cupierunt ne dicam venerari et adorare ostenderunt. Nam omnes quidem ita ad eius cineres ossaque certatim confluebant ut foelix et perbeatus is fore existimaretur qui ea videre et tangere foeliciorque tamen qui minimam ex illis particulam vendicare sibi posset quam alii argento auro alii recondere atque exornare faciebant et ad collum appendebant sibi tanquam rem divinam sanctam, et fatalem, et summa veneratione ac religione observabant existimantes illos omnes qui eas secum relliquias ferent, consimili quoque fortuna et foelicitate in vita usuros esse qua ipse Scanderbegus a Diis immortalibus impetrata solus ex omni hominum memoria dum viveret semper usus est. (HS, XIII, f. CLIX ${ }^{v}$ )

Como puede observarse, el encuentro ante el cuerpo no tiene por objeto una «damnatio memoriae» (Rader, 2006), ya que no se trata con escarnio, sino con temor y respeto. Los turcos afluyen masivamente para ver el cadáver de su ilustre enemigo, cuyo solo nombre causaba pavor («auditum nomen fugiebant»), con el fin de ver («videre») e incluso tocar sus restos («tangere») no para su veneración, como si se tratase de un cuerpo sagrado, sino como manifestación de un rito ancestral según el cual al tocar el cuerpo se conjura a su poseedor y se evita su reencuentro tras la muerte (Roux, 1984). De igual modo, la adquisición de partes del mismo ( «minimam

26. Ahora bien, la muerte de Scanderbeg, aunque líder de un pueblo, no debe entenderse como la de un rey, en quien su final lo es solo del cuerpo corruptible, pues permanece el cuerpo institucional (el cuerpo místico), que se transmite a la posteridad mediante la continuidad de la dinastía en tanto que expresión del cuerpo político. Véase Kantorowicz (2016). 
[...] particulam»), su posesión y uso, a modo de «amuleto»o «talismán» («posset quam alii argento auro alii recondere atque exornare faciebant et ad collum») se perciben como fragmentos que preservan y transmiten las cualidades del difunto ( $<$ consimili quoque fortuna et foelicitate in vita usuros esse qua ipse Scanderbegus $\gg)$, pues se consideran «representación» del mismo $(\ll \text { representatio» })^{27}$, al igual que las reliquias que contienen la esencia de un cuerpo santo, y diseminan el influjo de su «condición sobrenatural», de la que permiten participar a quienes entran en contacto con ellas o las poseen (Geary, 1990, Bynum, 2011 y Baschet, 2016) ${ }^{28}$.

\section{Conclusión}

La 'vida' de Jorge Castriota, Scanderbeg, elaborada por Marin Barleti más de un cuarto de siglo después de la muerte del noble albanés, en un momento en que el Imperio Otomano ha conseguido una enorme expansión y no deja de extenderse, y en que la Cristiandad, fragmentada, se siente amenazada, se articula siguiendo el modelo del miles Christi y se presenta como digna de imitación. Una 'biografía heroica' de un athleta Christi que, sin embargo, se distancia en parte del personaje histórico, quien especialmente combatió, por un lado, para tratar de preservar su patrimonio, al enfrentarse con otros albaneses, $y$, por el otro, para conservarlo y mantener las formas de vida albanesas, al hacerlo contra los turcos; y que no parece que hubiese concebido su lucha como una 'cruzada' contra el infiel.

Asimismo, es necesario destacar que la obra de Barleti devendrá canónica y que, en espacios y circunstancias distintos, que, sin embargo, compartirán rasgos de semejanza, será objeto de versiones y reelaboraciones diversas en las que esos «momentos» se adecuarán a sus varios contextos, ya sea en el ámbito de la escritura o en el de la oralidad. De ese modo, pervivirá en la 'memoria' la figura heroica de Scanderbeg desde su creación hasta hoy ${ }^{29}$.

\section{Bibliografía}

Aeneae Sylvil Piccolominei (1551), «De Europa $\gg$, Aeneae Sylvii Piccolominei Senensis, qui post adeptum Pontificatum Pius eius nominis secundus appellatus est Opera quae extant omnia, Basilea, Henrichum Petri, fols. 387-471.

BARLetius, Marinus (1508-1510), Historia de vita et gestis Scanderbegi Epirotarum Principis, Roma, B[ernardus] V[italis].

BARó I QUERALT, Xavier (2019), «Skanderbeg (1405-1468), heroi nacional albanès, entre la realitat i el mite», Temps d'Educació (Universitat de Barcelona), 56, pp. 221-234.

BAschet, Jerôme (2016), Corps et âmes. Une histoire de la personne au Moyen Âge, Paris, Flammarion.

Boulton, D’Arcy Jonathan Dacre (1987), The Knights of the Crown. The Monarchical Orders of Knighthood in Later Medieval Europe, 1325-1520, Woodbridge, The Boydell Press.

Brackob, A. K. (2018), Scanderbeg. A History of George Castriota and the Albanian Resistance to Islamic Expansion in Fifteenth Century Europe, Buffalo-Las Vegas-Palm Beach, Histria Book.

27. Empleo el término tal y como lo hace Ginzburg (1998).

28. En el folclore albanés ha pervivido la tradición de que después de su muerte el pueblo lo desentierra para fabricarse talismanes con sus huesos.

29. Para una visión general, véase Baró i Queralt (2019). 
Burrow, J[ohn] A[nthony] (1986), The Ages of Men. A Study in Medieval Writing and Thought, Oxford, Clarendon Press.

Bynum, Caroline Walker (2011), Christian Materiality. An Essays on Religion in Late Medieval Europe, New York, Zone Books.

Campbell, Joseph (1949), The Hero with a Thousand Faces, New York, Bollingen Foundation.

Charbonneau-Lassay, Louis (2006) [1941], Le Bestiaire du Christ, Paris, Albin Michel.

Colunga, Alberto y Laurentio Turrado (ed.) (1953), Biblia sacra iuxta Vulgatam Clementiam. Nova Editio, Matriti, Editorial Católica.

Contreras Martín, Antonio (2017), «Momentos estelares en la Vita de Carlos IV de Bohemia: poética y política», Estudis sobre pragmática de la literatura medieval / Estudios sobre pragmática de la literatura medieval, ed. Gemma Avenoza, Meritxell Simó, María Lourdes Soriano Robles, València, Universitat de València, pp. 105-117.

Flori, Jean (1986), L'essor de la chevalerie. XII ${ }^{e}$-XIII siècles, Genève, Droz.

Geary, Patrick, J. (1991), Furta Sacra: Theft of Relics in the Central Middle Ages, Princeton, Princeton University Press.

Genesin, Monica, Joachin Matzinger y Giancarlo Vallone (2010), The Living Scanderbeg. The Albanian Hero between Myth and History, Hamburg, Verlag Dr. Kovač.

Ginzburg, Carlo (1998), «Rappresentazione. La parole, l'idea, la cosa», en su Occhiacci di legno. Nove riflessioni sulla distanza, Milano, Feltrinelli, pp. 82-99.

Greenfield, Amy Butler (2005), A Perfect Red, New York, Doubleday.

Guglielmi, Nilda (ed.), (2002), El Fisiólogo, Madrid, Ediciones Eneida.

Hod gkinson, Harry (2004), Scanderbeg (edited by Bejtullah Destani \& Westrow Cooper; with an introduction by Dr. David Abulafia), London-New York, I. B. Tauris.

Hopf, Charles (1873), «Breve memoria di li discendenti de casa Musachi», en Chroniques grécoromanés inédites ou peu connues, Berlin, Librairie de Weidmann, pp. 240-340.

IsAku, Skënder, «L'art militaire de Skanderbeg», Studia Albanica, 5/1, pp. 169-176.

Kantorowicz, Ernst H. (2016), The King's Two Bodies: A Study in Medieval Political Theology, introd. Conrad Meyer, prol. William Chester Jordan, Princeton, Princeton University Press.

Keen, Maurice (1984), Chivalry, New Haven-London, Yale University Press.

Konstantin Michailović di Ostrovica (2001), Cronaca turca ovvero Memorie di un giannizzero, ed. Alda Giambelluca Kossova, Angiolo Danti, Marco Clementi, Palermo, Sellerio Editore.

KRITOvoulos (1954), History of Mehmet the Conqueror, trans. Charles T. Riggs, Princeton, Princeton University Press.

Laporta, Alessandro (2004), La vita di Scanderbeg di Paolo Angelo. Un libro anonimo restituito al suo autore, Galatina, Mario Congedo Editore.

Llull, Ramon (1988). Llibre de l'orde de cavalleria, ed. Albert Soler i Llopart, Barcelona, Barcino. Malaxecheverría, Ignacio (ed.) (1986), Bestiario medieval, Madrid, Siruela.

(1987), «El drac en el bestiari medieval», El drac en la cultura medieval, Barcelona, Fundació Caixa de Pensions, pp. 47-62.

March, Pere (1993), Obra completa, ed. Lluís Cabré, Barcelona, Barcino.

MichA, Alexander (ed.) (1978-1982), Lancelot, roman en prose du XIII siècle, Genève, Droz.

Montaner Frutos, Alberto (2013), El señal del rey de Aragón: historia y significado, Zaragoza, Institución «Fernando el Católico» [reimpresión electrónica corregida] https://ifc.dpz.es/ recursos/publicaciones/19/73/_ebook.pdf [14/04/2019] 
Nadin, Lucia (2012), Albania ritrovata, Tirana, Onufri.

Noli, Fan Stilian (1993), Scanderbeg, Lecce, Argo.

Orme, Nicholas (1984), From Childhood to Chivalry. The Education of the English King and Aristocracy, London, Routlegde.

RAder, Olaf B. (2006), Tumba y poder. El culto político a los muertos desde Alejandro Magno hasta Lenin, trad. María Condor, Madrid, Siruela.

Riché, Pierre y Danièle AleXandre-Bidon (1994), L'enfance au Moyen Age, Paris, Seuil.

Riquer, Martí de (2011), L'arnès del cavaller. Armes i armadures catalanes medievals, Barcelona, La Magrana.

Roux, Jean-Paul (1984), La religion des Turcs et Mongols, Paris, Payot.

Russell, Jeffrey Burton (1984), Lucifer: The Devil in the Middle Ages, Ithaca (New York), Cornell University Press.

Sabellicil, Marco Antonii (1556), Historiae Rerum Venetarum ab urbe condita, libri XXXIII, Basilea, Episcopus [Ejemplar Bayerische StaatsBibliothek Gs. 8262]. https://reader.digitalesammlungen.de/de/fs1/object/display/bsb11249872_00003.html [Consulta: 08/01/2020].

SANMARTÍn BASTIDA, Rebeca (2006), El arte de morir. La puesta en escena de la muerte en un tratado del siglo XV, Madrid-Frankfurt am Main, Iberoamericana-Vervuert.

Schama, Simon (1995), Landscape and Memory, London, Harper Collins.

Seadlar, Jean W. (2015), East Central Europe in the Middle Ages, 1000-1500, Washington, University of Washington Press.

Stein, Mark L. (2007), Guarding the Frontier. Ottoman Border Forts and Garrisons in Europe, London-New York, I. B. Tauris.

Sтоск, Markus (ed.) (2016), Alexander the Great in the Middle Ages: Transcultural Perspectives, Toronto-Buffalo-London, University of Toronto Press.

TuAn, Yi-Fu (1974), Topophilia. A study of enviromental perception, attitudes and values, Englewood Cliffs (New Jersey), Prentice-Hall.

Vaccaro, Attilio (2013), Studi storici su Giorgio Castriota Scanderbeg. Eroe cristiano albanese nella guerre antiturca, Lecce, Argo.

Zambon, Francesco (ed.) (1982), Il Fisiologo, Milano, Adelphi Edizione.

Zeqo, Epidamn (2017), «Gentile Bellini’s link with Albania in the Fifteenth Century and George Kastrioti Skanderbeg», New Eastern Europe (June 2). http://neweasterneurope. eu/2017/06/02/gentile-bellini-s-link-with-albania-in-the-fifteenth-century-and-george-kastrioti-skanderbeg/ [Consulta: 08/01/2021] 